\title{
Effects of training on postural control and agility when wearing socks of different compression levels
}

\author{
Timo Jaakkola ${ }^{1}$, Vesa Linnamo ${ }^{1}$, Mei Teng Woo ${ }^{1,2}$, Keith Davids ${ }^{3}$, Jarmo M. Piirainen ${ }^{1}$, \\ Arto Gråstén ${ }^{1,4}$, Jarmo Liukkonen ${ }^{1}$ \\ ${ }^{1}$ Faculty of Sport and Health Sciences, University of Jyväskylä, Finland; ${ }^{2}$ School of Sports, Health and Leisure, Republic \\ Polytechnic, Singapore; ${ }^{3}$ Centre for Sports Engineering Research, Sheffield Hallam University, UK; ${ }^{4}$ Faculty of Education, \\ University of Tasmania, Australia
}

\section{Summary}

Study aim: The aim of this study was to evaluate the effects of training while wearing socks differing in compression level (clinical, sub-clinical, regular) on performance of static and dynamic balancing and agility tasks in healthy, physically active people. We sought to understand whether socks with different compression properties supported postural regulation and agility task performance by enhancing somatosensory perception, unskewed by specific age range effects.

Material and methods: Participants comprised 61 adults aged 18-75 years, divided into three groups (two experimental groups wearing clinical or sub-clinical level compression socks, and one control group wearing regular non-compression socks during training). An 8 -week $(2 \times 1 \mathrm{~h}$ per week) intervention programme was administered to train static and dynamic balance and postural control, leg strength and agility.

Results: A mixed model ANOVA revealed no differences in static and dynamic balance and postural control and agility performance between clinical, sub-clinical, and control groups before and after training. All groups significantly improved their test performance, suggesting that training had some benefit on motor performance.

Conclusions: These results raised interesting questions requiring further investigation to examine the effects of wearing socks (with and without different levels of compression) on motor behaviours in specific groups of elderly vs. young participants, in physically active vs. less physically active people, and in performance settings outside standardized laboratory tests to study applications in natural performance environments.

Keywords: Postural control - Agility - Training intervention - Socks - Compression

\section{Introduction}

Compression-based garments represent a significant trend in top-tier sports $[1,6]$. Use of compression materials in garments was originally proposed to enhance physiological recovery from exercise, prevent injuries, and reduce chafing $[6,18]$. More recently, some evidence suggests that compression materials can enhance skill performance, possibly by mediating participant access to proprioceptive information for coordination of movement and balance control $[14,15,36]$. These data are aligned with findings from a pilot study by Pearce et al. [26] demonstrating positive motor performance (visuomotor tracking) whilst wearing compression garments during and after repetitive eccentric arm exercise. In more challenging conditions involving visual occlusion, compression garments (shorts) improved the total time in unipedal standing balance [21]. For young athletes with lower extremity injuries, studies have shown that compression via orthoses and sleeves improved uni - and bi-pedal balance performance $[2,10,19,22,23]$.

Currently, the evidence is somewhat mixed on the role of compression material in motor performance. For example, Bernhardt and Anderson [4] reported that wearing compression shorts did not enhance joint position sense in young adults. Furthermore, Papadapoulos et al. [24] suggested that ankle braces with $30 \mathrm{kPa}$ and $60 \mathrm{kPa}$ pressure were not able to alter the balance control strategy of the central nervous system (CNS). In an elderly sample, localized compression on the ankle was shown to improve joint position sense, but not static balance performance [16]. 
Here, we examined the possibility that the rather mixed findings may have emerged in the literature because wearing compression materials may need to be complemented with a training intervention to maximise benefits in enhancing somatosensory regulation of movements.

What might be the mechanism behind the enhancement of proprioceptive information from wearing such external attachments and garments? The tactile and proprioceptive sub-systems play a role in regulating balance by responding to information from fine touch, compression, contortion, vibration, pressure and changes in joint angles that is picked up by somatosensory system components [19]. One possibility is that external appliances (e.g. tapes, braces, neoprene sleeves, compression garments) could contort, compress and stimulate muscle and soft tissue to enhance the level of sensorimotor system noise, thus providing better neuromuscular control and performance [5]. Through this continuous process of tissue contortion and compression, it is assumed that compression garments might provide additional somatosensory information to the skin, muscle and joint mechanoreceptors of the feet, which can help in regulating posture and enhance agility $[14,36]$.

Furthermore, despite the emerging evidence on its role in enhancing skill performance, previous research has not yet determined whether compression garments need to be at a clinical level to facilitate skill performance through enhancing attunement to proprioception [14, 15]. Additionally, previous research has mainly focused on the role of compression garments in sport performance. These studies have mainly investigated immediate (perhaps temporary) effects of wearing compression garments in elite athletic samples. For example, such research has investigated elite alpine skiers [32], female athletes [21], and young athletes with lower limb injuries $[2,10,23,38]$. Investigations on the impact of wearing compression garments, such as socks, on proprioception to regulate motor performance have been infrequent, with non-athletic samples. A related issue concerns whether compression material in socks can enhance motor performance in nonathletic populations. It remains unclear whether wearing textured compression socks, at a clinical or sub-clinical level, may benefit balance control and agility performance in ordinary people during their daily living activities. This issue is important since it needs to be understood whether wearing compression garments only benefits from attunement to proprioceptive information in skilled athletes due to their extensive use of proprioceptive information during sports training [13]. For this reason, we considered that it might be useful to study the integration of a training intervention to enhance attunement to proprioceptive information in ordinary people, along with the use of compression materials with different properties, to understand the effects on motor performance, such as when balancing and regulating posture.
Postural and dynamic balance control are important to most normal daily activities and complex motor skills [7]. In the elderly population, decline in balance control is a major risk factor for falls [3]. In sports, the standing limb in the kicking action in soccer and taekwondo is important to ensure stability needed for successful execution $[25,35]$. From an injury prevention perspective, balance deficit that occurs immediately after anaerobic stress and fatigue could affect sports performance and might increase the probability of injuries $[29,32]$.

In this study, we sought to consider whether use of compression socks at a clinical (compression level between 20 and $40 \mathrm{mmHg}$; Zeropoint, Finland) or sub-clinical level (below $20 \mathrm{mmHg}$ ) would influence the performance in balance and agility tasks in a sample of adults who were not elite athletes, but were physically active. It is relevant to understand whether there may be a cross-over effect of compression technology in aiding the motor performance of people without extensive experience of training in sport. Given the lack of clarity in existing research, the specific purpose of this study was to investigate the effectiveness on participant balance and agility performance of wearing socks, differing in clinical and sub-clinical levels, in comparison to performance with a control group wearing regular socks, in an eight-week training intervention.

We sought to observe whether differences in compression levels in knee length socks (clinical, sub-clinical and non-compression) would help participants achieve better balance control and agility performance, in conjunction with a training intervention. We sought to understand whether experimental groups, wearing clinical-level and sub-clinical-level compression socks, would improve dynamic and static balance performance and agility more during the intervention. We also expected training to interact with the sock properties to support task performance in the physically active participants.

\section{Materials and methods}

\section{Participants and setting}

Study participants were recruited by advertisements in a local newspaper and on the University of Jyväskylä internet homepage. A wide-ranging sample of adults was sought, with participants comprising 61 adults (54 females, 7 males) between the ages of 18 and 75 years. Their experience with physical activity and medical condition were examined by a written survey, and those with severe health problems were excluded for health and safety reasons. They were selected as a non-athletic sample, defined as not being physically inactive, yet not classified as skilled or elite in a particular sport and physical activity. Participants were next ranked from lowest to highest based on the dynamic balance centre of pressure (COP) area $\left(\mathrm{mm}^{2}\right)$ 
observed during a 1-minute assessment, which consisted of implementing eight balance perturbations in the anterior and posterior direction. COP area was chosen as the dependent variable, because it is a common measure reported in the balance literature $[27,28]$, is available immediately after recording, and did not need any further data analysis. Thereafter, participants were divided into two groups based on initial COP area results (highest to lowest) and 50\% were randomly allocated to the test group and $50 \%$ to the control group. Mean age of the clinical experimental group was 53.8 years (sd $17.0 ; \mathrm{n}=25)$ and of the sub-clinical experimental group 45.9 years (sd 15.8; $\mathrm{n}=25)$. In order to analyse whether compression, at clinical or non-clinical levels, would be beneficial along with the training, we also performed the test protocol and the intervention with an additional control group wearing regular, non-compression socks. The mean age of this additional control group was 46.6 years ( $\mathrm{sd} 12.0 ; \mathrm{n}=11)$. Originally the control group comprised 18 participants, but seven dropped out or did not participate to the end of the measurements. One-way ANOVA revealed no significant age differences between the clinical experimental, sub-clinical experimental, and control group $\left(F_{(2,60)}=1.767, p=0.180\right)$. Characteristics of participants are presented in Table 1.

The mean value of the COP area parameter was 3564 (sd 1729$) \mathrm{mm}^{2}$ for the clinical experimental group, 3552 (sd 1359 ) $\mathrm{mm}^{2}$ for the sub-clinical group, and 7352 (sd 3 272) $\mathrm{mm}^{2}$ for the control group. Analysis showed no statistically significant differences between the clinical and sub-clinical groups $\left(F_{(1,49)}=0.001, p=0.978, d=0.01\right.$, $95 \%$ CI $[-168.52,192.52])$, but significant differences between the clinical and control group $\left(F_{(1,35)}=20.842\right.$, $p<0.001, d=-1.45,95 \% C I[3321.33,4254.67])$ and the sub-clinical and control group $\left(F_{(1,35)}=24.774, p<.001\right.$, $d=-1.52,95 \%$ CI $[3353.22,4246.78])$ were found.

Participants were asked to sign a written informed consent form and were informed of the protocol and possible risks of the study. They were also advised of their right to withdraw from the study at any time. The study was conducted according to the Declaration of Helsinki and approved by the University of Jyväskylä ethics committee.

\section{Intervention}

The experimental (clinical and sub-clinical) and the control group participants were mixed gender, exercising in three groups, for two one-hour, supervised sessions per week, for a period of eight weeks in a university gymnasium. All groups had identical training sessions, which consisted of a variety of static and dynamic balance exercises as well as exercises that improve kinaesthetic sense and agility. Each session started with a standardized 10-minute warmup including slow-paced running and stretches. The main exercise phase lasted 40 minutes and included the following structure: a) 20 minutes of balance exercises including, for example, balancing and postural control training units, such as the Romberg stance, one - and two-legged stances with eyes open and closed, dynamic balance training landing on one leg and two legs on different surfaces, resistance training of the lower limbs, static and dynamic balancing on beams and uneven surfaces, and on soft surfaces and mattresses, circuit training workout designed to challenge balance skills, and squats on a balance board; b) 20 minutes of agility exercises, including, for example, ladder run exercises, an exercise course constructed of buckets filled with bean bags to be moved to different points of the gym, jump rope, bench jump, and jumping jacks. The training session ended with a standardized 10-minute cool-down including stretches. The programme and individual exercises were designed in collaboration with the research team and took safety issues into account.

Participants were not aware of belonging to a clinical, sub-clinical or control group. The former group used clinical level compression socks during training. Sock size was individualized, based on shoe size as well as ankle and calf circumferences. The control group participants wore socks of similar size, thickness, and outlook, but without clinical levels of compression, individually chosen by shoe size only. Participants used the compression socks only during the intervention periods in the training sessions. Their physical activity outside the intervention was recorded using a training diary. Analysis of weekly lightto-vigorous hours of physical activity changed into mean $\mathrm{MET} / \mathrm{min}$ values per day, based on self-report diaries

Table 1. Characteristics of subjects

\begin{tabular}{lccc}
\hline Variable & Clinical group & Sub-clinical group & Control group \\
\hline $\mathrm{N}$ & 25 & 25 & 11 \\
Males/females & $2 / 23$ & $2 / 23$ & $1 / 10$ \\
$\mathrm{Age}(\mathrm{SD})$ & $53.8(17.0)$ & $45.9(15.8)$ & $46.6(12.0)$ \\
$\mathrm{COP}(\mathrm{SD}) \mathrm{mm}^{2}$ & $3564(1729)$ & $3552(1359)$ & $7352(3272)$ \\
Physical activity - MET/min per day (SD) & $275.67(78.66)$ & $274,50(66.46)$ & $284,83(50.49)$ \\
\hline
\end{tabular}

$\mathrm{COP}=$ center of pressure measured before intervention. Physical activity=physical activity engagement before intervention from participants' diaries. 
outside the training programme during the intervention period, showed no differences between the two experimental groups and the control group (ANOVA; $p=0.914$ ).

The clinical compression socks used in this study were made by Zero Point Finland (https://www.zp.fi). The materials consisted of $20 \%$ nylon, $20 \%$ Lycra and $60 \%$ Coolmax. The socks that were used were the Intense Compression Socks, which are also the most compressive $(20-30 \mathrm{mmHg})$ sock product the company offers. The socks were designed to have a graduated compression that was tighter around the ankle while decreasing the compression closer to the knee. The sub-clinical group used compression socks made by Pro Touch (http://www.protouch.eu), made of $98 \%$ polyamide and 2\% Elastane, and selected for testing because they did not have graduated compression from the ankle to the calf. The control group wore regular socks. Pre- and posttests were undertaken before and after the training intervention treatment programme.

\section{Measures}

Dynamic balance. A dynamic test on a force platform was performed because it can separate poor and good balance control more efficiently than a static balance test [27]. Dynamic balance was measured using a commercial force plate (BT4 balance platform, HurLabs Oy, Tampere, Finland), which was placed over a typical perturbation device (University of Jyväskylä, Finland). The perturbation device consisted of two steel frames (bottom $910 \times 910 \mathrm{~mm}$, middle $910 \times 610 \mathrm{~mm}$ ), constructed from four $60 \times 30 \mathrm{~mm}$ steel tubes. A steel plate was placed over the middle frame, upon which sat the force plate. Wheels were placed under the middle frame and the upper steel plate, enabling sideways movement of the middle plate and antero-posterior movement of the upper steel plate. Two electromechanical cylinders (EMC, Bosch Rexroth, Germany) produced movement of the frames with a maximal amplitude of $300 \mathrm{~mm}$, maximal force of $4000 \mathrm{~N}$, and maximal velocity of $70 \mathrm{~cm} / \mathrm{s}$. The cylinders were connected to three-phase motors (MSK030B, Bosch Rexroth, Germany) and frequency encoders (HCS02, Bosch Rexroth, Germany), which were controlled via an NI 4-channel analogue card (NI 9263, National Instruments, USA) and NI 8-channel digital card (NI 9472, National Instruments, USA). Both cards were connected to a USB card (NI cDAQ-9172, National Instruments, USA), which was connected to the computer. Perturbation parameters (acceleration, velocity, amplitude) were determined and delivered manually using Labview (National Instruments, USA) and IndraWorks software (Bosch Rexroth, Germany).

For dynamic balance measurement the system moved in the horizontal direction with the two following conditions: maximum acceleration $0.5 \mathrm{~m} / \mathrm{s}^{2}$ and $2.5 \mathrm{~m} / \mathrm{s}^{2}$, maximum velocity $15 \mathrm{~cm} / \mathrm{s}$ and $25 \mathrm{~cm} / \mathrm{s}$ in slow and fast perturbations, respectively. Plate displacement was at a constant level of $15 \mathrm{~cm}$ in all conditions. In total, 4 balance perturbations were delivered in both anterior (plate moved forward) and posterior (plate moved backward) directions, in 6- to 8-second intervals in a random order during a 1-minute period in both conditions. A black mark was fixed on the wall $12.8 \mathrm{~m}$ from the participant at eye level in order to stabilize the visual focus during the measurements. In the study of Piirainen et al. [29] COP maximal displacement in the anterior direction showed the greatest differences between young and elderly participants. This parameter was therefore chosen for further analysis also in the present study and the average of four perturbations was calculated from the anterior direction with the two speeds, fast anterior perturbation (FAP) and slow anterior perturbation (SAP). Participants used a safety harness to avoid any falling accidents.

Agility. The figure-of-eight run [34] test was used to analyse participants' agility. The figure-of-eight track was marked with two cones placed $10 \mathrm{~m}$ apart with the start/ finish line next to one of the cones, which ultimately formed a $20 \mathrm{~m}$ long track. The stopwatch was started with the starting signal, and the participant ran to the second cone as fast as possible, circled it, and returned to the starting line around the first cone. The stopwatch was stopped when the participant crossed the starting line again. The time was recorded in seconds. One practice trial was allowed, and the test was performed twice with a short rest between each trial. The better result was the participant's final score.

Static balance. The study used the flamingo standing test [8] to examine the balance skills of the population. The test measures especially the static balance, because it is performed standing still. In this test the participant stood on a 50-cm long, 4-cm high, $3-\mathrm{cm}$ wide wooden beam for 60 seconds on one leg while holding the other leg up. The free leg was bent backwards and the back of the foot was gripped with the hand on the same side. Switching the leg was allowed. Each time the participant lost their balance by releasing the free leg or touched the floor with any part of the body, the stopwatch was stopped. After each such fall, the same procedure was started again. The number of attempts required within the $60 \mathrm{~s}$ time period was the participant's final score.

\section{Data analysis}

First, the normal distribution, missing values, and outliers were examined. No modifications due to the distribution or outliers were required. Missing values comprised $12.3 \%$ of the data. Little's MCAR test $\chi^{2}=23.421, d f=30$, $p=0.798)$ revealed that the missing values were not systematic, and thus the values were assumed to be missing completely at random (MCAR) [20]. Next, means and standard deviations were calculated. Finally, mixed between-within subjects ANOVA (analysis of variance) with the Scheffé post hoc test was used to compare test scores between clinical, sub-clinical, and control groups across 
pre- and post-measurement in order to analyse possible interactions. Because Levene's test of equality of error variances $(p<0.05)$ and Box's test of equality of covariance matrices $(p<0.001)$ were violated through the pre scores of the flamingo standing test, an ANCOVA (analysis of covariance) was performed to adjust the existing statistical difference between clinical, sub-clinical, and control groups. Results were considered statistically significant at the alpha level of $p<0.05$. Data were analysed using PASW software version 22.0 (Armonk, NY: IBM Corp).

\section{Results}

Pearson product moment correlations showed statistically significant, positive associations between the flamingo standing score and figure-of-eight run score, the flamingo standing score and FAP, as well as the figure-ofeight run score and FAP (Table 2). Descriptive statistics of the study variables are presented in Table 3 .

A mixed model analysis of variance was conducted to compare the SAP scores between clinical, sub-clinical, and control groups across two time points. The results showed no statistically significant interaction in SAP between clinical, sub-clinical, and control groups and pre- and post-

Table 2. Correlations among study variables $(n=61)$

\begin{tabular}{lccc}
\hline Variable & 1 & 2 & 3 \\
\hline 1. SAP (slow anterior perturbation) & - & & \\
2. FAP (fast anterior perturbation) & 0.25 & - & \\
3. Flamingo standing test & 0.01 & $0.41^{*}$ & - \\
4. 8-run test & -0.05 & $0.37^{*}$ & $0.70^{* *}$ \\
\hline
\end{tabular}

$*-\mathrm{p}<0.01, * *-\mathrm{p}<0.001$. measurement (Wilks' lambda $=0.987, F_{(2,48)}=0.327$, $p=0.722$, partial eta squared $=0.013$ ). However, the results showed a significant main effect for pre- and post-measure (Wilks' lambda $=0.875, F_{(1,48)}=6.881, p=0.012$, partial eta squared $=0.125$ ), with all groups showing a decrease in SAP during the intervention. Subsequently, the main effect comparing clinical, sub-clinical, and control groups was significant $\left(F_{(2,48)}=10.802, p<0.001\right.$, partial eta squared $=0.310)$, suggesting significant differences between groups. The Scheffé post hoc test revealed significant differences in SAP between the pre-measurements of the clinical and control group $(p<0.05)$ and the postmeasurements of the clinical and control group $(p<0.001)$ as well as the sub-clinical and control group $(p<0.001)$.

The results indicated that there was no significant interaction effect on FAP between the clinical, sub-clinical, and control group and pre- and post-measurement (Wilks' lambda $=0.976, F_{(2,48)}=0.591, p=0.558$, partial eta squared $=0.024)$. In contrast, the model showed a significant main effect for pre- and post-measurement (Wilks' lambda $=0.787, F_{(1,48)}=12.996, p=0.001$, partial eta squared $=0.213$ ) with all groups showing a decrease in FAP scores during the intervention. In addition, the main effect comparing clinical, sub-clinical, and control groups was not statistically significant $\left(F_{(2,48)}=1.074, p=0.350\right.$, partial eta squared $=0.043)$, showing no effect on FAP scores between groups. However, Table 3 shows that the percentage improvement in FAP scores was over twice as much in the clinical compression group compared to the other groups, although the effect size was small.

The model results demonstrated that there was no significant interaction in figure-of-eight runs between clinical, sub-clinical, and control groups and pre- and post-measurement (Wilks' lambda $=0.997, F_{(2,45)}=0.077, p=0.926$, partial eta squared $=0.003$ ). However, the results showed a significant main effect for pre- and post-measurement

Table 3. Descriptive statistics of the study variables

\begin{tabular}{|c|c|c|c|c|}
\hline Group & Variable & Pretest $(\mathrm{M} \pm \mathrm{SD})$ & Posttest $(\mathrm{M} \pm \mathrm{SD})$ & Change $[\%]$ \\
\hline \multirow{4}{*}{$\begin{array}{l}\text { Experimental group }(\mathrm{n}=25) \\
(\text { Clinical })\end{array}$} & SAP (slow anterior perturbation) & $67.72 \pm 11.47)$ & $63.79 \pm 6.12)$ & -5.80 \\
\hline & FAP (fast anterior perturbation) & $121.70 \pm 41.80)$ & $99.32 \pm(35.37)$ & -18.39 \\
\hline & Flamingo standing test & $24.91 \pm 10.38)$ & $18.00 \pm 7.72)$ & -27.74 \\
\hline & 8 -run test & $8.72 \pm 1.38)$ & $8.20 \pm 1.14)$ & -5.96 \\
\hline \multirow{4}{*}{$\begin{array}{l}\text { Experimental group }(\mathrm{n}=25) \\
(\text { Sub-clinical })\end{array}$} & SAP (slow anterior perturbation) & $71.66 \pm 13.51)$ & $65.62 \pm(9.45)$ & -8.43 \\
\hline & FAP (fast anterior perturbation) & $113.77 \pm 33.87)$ & $107.77 \pm 38.22)$ & -5.27 \\
\hline & Flamingo standing test & $15.15 \pm 9.25)$ & $13.65 \pm 9.21)$ & -9.90 \\
\hline & 8 -run test & $8.24 \pm 1.44)$ & $7.73 \pm 1.14)$ & -6.19 \\
\hline \multirow{4}{*}{$\begin{array}{l}\text { Control group }(\mathrm{n}=11) \\
\text { (Regular) }\end{array}$} & SAP (slow anterior perturbation) & $82.21 \pm 9.10)$ & $79.66 \pm 12.69)$ & -3.10 \\
\hline & FAP (fast anterior perturbation) & $133.25 \pm 32.94)$ & $120.92 \pm 23.22)$ & -9.25 \\
\hline & Flamingo standing test & $15.09 \pm 2.95)$ & $11.64 \pm 5.80)$ & -22.86 \\
\hline & 8 -run test & $7.52 \pm 0.80)$ & $7.14 \pm 0.77)$ & -5.05 \\
\hline
\end{tabular}


(Wilks' lambda $=0.467, F_{(1,45)}=51.434, p=0.000$, partial eta squared $=0.533$ ), with all groups showing a decrease in figure-of-eight run scores during the intervention. Additionally, the model demonstrated that the main effect comparing clinical, sub-clinical, and control groups was not significant $\left(F_{(2,45)}=3.073, p=0.056\right.$, partial eta squared $=0.120$ ), showing no difference in figure-of-eight run scores between the three groups.

Finally, the interaction effect on the flamingo standing test result between clinical, sub-clinical, and control groups across pre- and post-measurement was investigated. The results of ANCOVA demonstrated that after controlling for the baseline score, there were no statistically significant differences between clinical, sub-clinical, and control groups in the flamingo standing test score $\left(F_{(2,47)}=1.768, p=0.183\right.$, partial eta squared $\left.=0.074\right)$. However, the results showed that after adjusting for the initial differences, the main effect for the flamingo test pre-score was significant $\left(F_{(1,47)}=66.043 p=0.000\right.$, partial eta squared $=0.600)$.

Taken together, all groups showed a decrease in SAP, FAP, and figure-of-eight run scores across the intervention. The baseline scores of SAP were lower among the clinical group than the control group, whereas the followup scores were lower among both clinical and sub-clinical groups compared to the control group. In contrast, the clinical group had higher figure-of-eight run follow-up scores than the control group. The baseline scores of the flamingo standing test predicted the follow-up scores, when the group membership was not linked with the follow-up scores.

\section{Discussion}

The purpose of this study was to investigate whether clinical and sub-clinical compression levels in knee length socks would provide improvements in performance in standard laboratory tasks for assessing balance and agility, compared to the control group, and whether clinical and sub-clinical compression levels would provide different levels of stimulation to lower leg mechanoreceptors, and enhance regulation of balance, postural control and agility performance in a wide-ranging sample of physically active people across the age range. The results of this study demonstrated that there were no differences in performance in standard laboratory tests of agility and balance between participants wearing clinical and sub-clinical compression socks, and a control group. Since regulation of balance and postural control in participants was enhanced by compression material in socks with different properties, the findings imply that even wearing sub-clinical level compression socks can enhance motor behaviour, when complemented with a training programme in physically active people.
Our findings implied that a sample of adults who were physically active, but not elite athletes, may have benefitted from wearing compression socks during a training intervention, regardless of whether the socks were clinical or sub-clinical in compression level. There were some statistically non-significant effects suggesting that wearing clinical or sub-clinical level compressions socks may have benefited the physically active participants (\% improvement in Table 3). These data warrant further investigations with more specific groupings such as elderly or young participants only or with physically inactive individuals. Clinical, sub-clinical, and control groups improved their dynamic balance control during the 8-week intervention, suggesting that training is an important aspect of enhancing postural control and agility performance in physically active people across the lifespan. It has been shown earlier that sensorimotor training can improve the rate of force development [11], which is an important characteristic in good and efficient balance control $[27,28]$. In the present study, we did not measure any force production properties, but the decreased agility test time in both groups indicates that some neuromuscular changes occurred. These changes may have also influenced the improvements observed in dynamic balance control. This is also supported by the positive correlation found between the figure-of-eight run score and FAP. On the other hand, Yaggie and Campbell [37] have shown that balance training in the young will lead to improved agility test scores. They did not, however, find any improvements in vertical jump performance, which might indicate that some other mechanism than the improved force production was involved in improving agility score and balance performance. One possible explanation, which needs to be tested in follow-up research, might be enhanced attunement to proprioceptive system feedback. This possibility might be excluded in future work by studying the performance of additional control group participants who simply wear socks of different compression levels and undergo test performance, without experiencing training interventions. It is noteworthy that Gruber et al. [12] reported reduced stretch reflex and H-reflex responses after balance training, which was suggested to be caused by increased pre-synaptic inhibition via increased supraspinal input. Both groups in their study improved their balance control and agility test time similarly, which indicates that additional compression did not lead to any specific improvement in balance control or performance.

It should also be noted that in this study, we did not control the effects of age by dividing the participants into age-related groups. We deliberately sought to study a wide-ranging sample of ordinary people who were not elite athletes. It has been suggested previously that the control strategy may change during ageing [9] which may lead to different neuromuscular responses during balance perturbations [29]. This may also cause some differences 
in training adaptations between young and elderly participants, which may explain the quite large variations in dynamic balance control in both pre- and post-tests. Nevertheless, this was not our objective in the present study, and it should be studied in more detail in future work. In the present study, it is difficult to speculate on possible changes in neuromuscular control, because none of those aspects were specifically measured. However, because no differences between the groups were observed, it can be speculated that the additional compression did not cause additional improvement in proprioception activity.

Another issue that needs to be examined is the length of the intervention period. The 8-week intervention training period may have been too short to reveal differences in balance and agility performance due to additional compression levels of socks worn by the experimental groups. Therefore in future, it is important to conduct longer interventions to investigate the interaction of different material properties on motor performance, including texture and compression levels (at clinical and sub-clinical levels). Although previous work has compared effects of wearing compression socks with barefoot conditions on motor performance, future experimental research should also investigate the effects of different compression levels with control conditions of no socks, for additional verification. Nevertheless, the findings of this study provided some tentative indications of equivalent effects of wearing clinical and sub-clinical compression socks, to contort muscle, skin and soft tissue of the lower leg and feet to enhance standing balance, postural control and agility task performance in a sample which is not composed of elite athletes.

\section{Conclusions}

The results of this study raised interesting questions requiring further investigation to examine the effects of wearing socks (with and without different levels of compression) on motor behaviours in specific groups of elderly vs. young participants, in physically active vs. less physically active people, and in performance settings outside standardized laboratory tests to study applications in natural performance environments.

\section{Conflict of interest: Authors state no conflict of interest.}

\section{References}

1. Akseki D., M. Erduran, K. Defne (2012) Sports Injuries and Proprioception: Current Trends and New Horizons in Sports Injuries. In: M.D. Doral (ed) Sports Injuries. Springer Berlin, Heidelberg, p. 67-71.
2. Baier M., T. Hopf (1998) Ankle orthoses effect on singlelimb standing balance in athletes with functional ankle instability. Arch. Phys. Med. Rehab., 79: 939-944.

3. Berg K.O., B.E.,Maki, J.I. Williams, P.J. Holliday, S.L. Wood-Dauphinee (1992). Clinical and laboratory measures of postural balance in an elderly population. Archiv. Physic. Med. Rehab., 73(11): 1073.

4. Bernhardt T., G.S. Anderson (2005) Influence of moderate prophylactic compression on sport performance. J. Strength Cond. Res., 19(2): 292-297.

5. Davids K., R. Shuttleworth, C. Button, I. Renshaw, P. Glazier (2003) "Essential noise" - enhancing variability of informational constraints benefits movement control: A comment on Waddington and Adams. Br. J. Sports Med., 38: 601-605.

6. Doan B.K., Y.-H. Kwon, R.U. Newton, J. Shim, E.M. Popper, R.A. Rogers, L.R. Bolt, M. Robertson, W.J. Kraemer (2003) Evaluation of a lower-body compression garment. J. Sports Sci., 21(8): 601-610.

7. Donath L., R. Roth, A. Rueegge, M. Groppa, L. Zahner, O. Faude (2013) Effects of slackline training on balance, jump performance and muscle activity in young children. Int. J. Sports Med., 34(12): 1093-1098.

8. EUROFIT (1988) European test of physical fitness. Rome: Council of Europe, Committee for the development of sport.

9. Fransson P.A., E.K. Kristinsdottir, A. Hafström, M. Magnusson, R. Johansson (2004) Balance control and adaptation during vibratory perturbations in middleaged and elderly humans. Eur. J. App. Physiol., 91(5-6): 595-603.

10. Genthon N., E. Bouvat, J.J. Banihachemi, J. Bergeau, A. Abdellaoui, P.R. Rougier (2010) Lateral ankle sprain alters postural control in bipedal stance: Part 2 sensorial and mechanical effects induced by wearing an ankle orthosis. Scan. Journal Med. Sci. Sports, 20: 255-261.

11. Gruber M., S.B. Gruber, W. Taube, M. Schubert, S.C. Beck, A. Gollhofer (2007a) Differential effects of ballistic versus sensorimotor training on rate of force development and neural activation in humans. J. Strength Cond. Res., 21(1): 274-282.

12. Gruber M., W. Taube, A. Gollhofer, S. Beck, F. Amtage, M. Schubert (2007b) Training-specific adaptations of $\mathrm{H}$ - and stretch reflexes in human soleus muscle. J. Mot. Behav., 39(1): 68-78.

13. Han J., G. Waddington, J. Anson, R. Adams (2015) Level of competitive success achieved by elite athletes and multi-joint proprioceptive ability. J. Sci. Med. Sport, 18: $77-81$.

14. Hasan H., K. Davids, J.Y. Chow, G. Kerr (2015) The Effects of Wearing Textured Insoles and Clinical Compression Socks on Kicking Performance in Association Football. Communication to World Congress in Science and Football, May 20-23, Copenhagen, Denmark. 
15. Hasan H., K. Davids, J.Y. Chow, G. Kerr (Under review) Compression and Texture in Socks Enhance Football Kicking Performance.

16. Hijmans J.M., W. Zijlstra, J.H.B. Geertzen, A.L. Hof, K. Postema (2009) Foot and ankle compression improves joint position sense but not bipedal stance in older people. Gait Post., 29: 322-325.

17. Hrysomallis C. (2007) Relationship between balance ability, training and sports injury risk. Sports Med., 37(6): 547-556.

18. Kraemer W.J., J.A. Bush, R.U. Newton, N.D. Duncan, J.S. Volek, C.R. Denegar, P. Canavan, J. Johnstone, M. Putukian, W.J. Sebastianelli (1998) Influence of a compression garment on repetitive power output production before and after different types of muscle fatigue. Sports Med. Train. Rehab., 8(2): 163-184.

19. Kuster M.S., K. Grob, M. Kuster, G.A. Wood, A. Graeme (1999) The benefits of wearing a compression sleeve after ACL reconstruction. Med. Sci. Sports Exerc., 31(3): 368-371.

20. Little R., D. Rubin (2002) Statistical analysis with missing data. New York, Wiley.

21. Michael J.S., S.N. Dogramaci, K.A. Steel, K.S. Graham (2014) What is the effect of compression garments on a balance task in female athletes? Gait Post., 39: 804-809.

22. Ozer D., G. Senbursa, G. Baltaci, M. Hayran (2009) The effect on neuromuscular stability, performance, multijoint coordination and proprioception of barefoot, taping or preventative bracing. The Foot, 19: 205-210.

23. Palm H.G., F. Brattinger, B. Stegmueller, G. Achatz, H.J. Riesner, B. Friemert (2012) Effects of knee bracing on postural control after cruciate ligament rupture. The Knee, 19: 664-667.

24. Papadopoulos E.S., C. Nikolopoulos, A. Badekas, G. Vagenas, S.A. Papadakis, S. Athanasopoulos (2007) The effect of different skin-ankle brace application pressures on quiet single-limb balance and electromyographic activation onset of lower limb muscles. BMC Musculoskel Disord., 8(89): 1-8.

25. Pau M., G. Ibba, G. Attene (2014). Fatigue-induced balance impairment in young soccer players. J. Athl. Train., 49(4): 454-461.

26. Pearce A.J., D.J. Kidgell, L.A. Grikepelis, J.S. Carlson (2009) Wearing a sports compression garment on the performance of visuomotor tracking following eccentric exercise: a pilot study. J. Sci. Med. Sport., 12: 500-502.

27. Piirainen J.M., J. Avela, N. Sippola, V. Linnamo V (2010) Age dependency of neuromuscular function and dynamic balance control. Eur. J. Sport. Sci., 10: 69-79.

28. Piirainen J., N.J. Cronin, J. Avela, V. Linnamo (2014) Effects of plyometric and pneumatic explosive strength training on neuromuscular function and dynamic balance control in 60-70 year old males. J. Electr. Kinesiol., 24: 246-252.
29. Piirainen J., V. Linnamo, N.J. Cronin, J. Avela (2013) Age-related neuromuscular function and dynamic balance control during slow and fast balance perturbations. J. Neurophysiol., 110: 2557-2562.

30. Roerdink M., P. Hlavackova, N. Vuillerme (2011) Centerof pressure regularity as a market for attentional investment in postural control: A comparison between sitting and standing postures. Hum. Mov. Sci., 30: 203-212.

31. Ruhe A., R. Fejer, B. Walker (2010) The test-retest reliability of centre of pressure measures in bipedal static task conditions - A systematic review of the literature. Gait Post., 32: 436-445.

32. Sperlich B., D.P. Born, M. Swarén, Y. Kilian, B. Geesmann, M. Kohl-Bareis, H.C. Holmberg (2013) Is leg compression beneficial for alpine skiers. BMC Sports Sci. Med. Rehab., 5(18): 1-12.

33. Steinberg N., D. Nemet, M. Pantanowitz, A. Zeev, M. Hallumi, M. Sindiani, Y. Meckel A. Eliakim (2016) Longitudinal study evaluating postural balance of young athletes. Percept. Mot. Skills, 122(I): 256-279.

34. Tegner Y., J. Lysholm, M. Lysholm, J.A. Gillquist (1986) A performance test to monitor rehabilitation and evaluate anterior cruciate ligament injuries. Am. J. Sports Med., 14(2): 156-159.

35. Woo M.T., J.Y. Chow, M. Koh (2014) Effect of Different Attentional Instructions on the Acquisition of a Serial Movement Task. J. Sports Sci. Med., 13: 782-792.

36. Woo M.T., K. Davids, J. Liukkonen, T. Jaakkola, J.Y. Chow (2014) Effects of textured compression socks on postural control in physically active elderly individuals. Proc. Engineering, 72: 162-167.

37. Yaggie J.A., B.M. Campbell (2006) Effects of balance training on selected skills. J. Strength Cond. Res., 20(2): 422-428.

38. You S.H., K.P. Granata, L.K. Bunker (2004) Effects of circumferential ankle pressure on ankle proprioception, stiffness, and postural stability: A preliminary investigation. J. Orthop. Sports Phys. Ther., 34: 449-460.

\section{Received 31.01.2017 \\ Accepted 30.06.2017}

(C) University of Physical Education, Warsaw, Poland

Acknowledgments

Authors want to thank Zero Point Finland for providing socks used in this study, as well as Master's students Daniel Kiikka, Miikka Sinko, and Jaakko Niemi-Nikkola for running the intervention practices.

None of the authors have any association with Zero Point Company and the authors have no conflict of interest. 